\title{
Activation of multiple Toll-like receptors serves different roles in sepsis-induced acute lung injury
}

\author{
XINLEI CHEN ${ }^{1}$, TINGTING WANG ${ }^{1}$, LIANG SONG ${ }^{2}$ and XIANGYAN LIU ${ }^{2}$ \\ Departments of ${ }^{1}$ Anesthesia and ${ }^{2}$ Thoracic Surgery, Shandong Provincial Hospital Affiliated to \\ Shandong University, Jinan, Shandong 250000, P.R. China
}

Received June 26, 2018; Accepted March 7, 2019

DOI: $10.3892 /$ etm.2019.7599

\begin{abstract}
The activation of Toll-like receptors (TLRs) is involved in the innate immune response and the acute inflammatory response following sepsis-induced acute lung injury (ALI). Increasing evidence has demonstrated that sepsis-induced ALI may be closely associated with several abnormal TLRs, activated by components of microorganisms. However, the number of TLRs involved in this process and the extent of their involvement has not been fully elucidated. The current study examined the simultaneous activation of four TLRs closely associated with sepsis-induced ALI. The results demonstrated that in contrast to the sham-operated group, the mRNA and protein expression levels of TLR2/4/9 were significantly increased in the cecal ligation and puncture (CLP)-operated group. In addition, TLR2 $2^{-/}$, TLR3 ${ }^{-/}$, $\mathrm{TLR}^{-/-}$and $\mathrm{TLR}^{-/-}$C57BL/6 mice were used to establish a CLP-induced ALI animal model and measure the expression levels of TNF- $\alpha$ and IL- 6 in plasma and lung tissue samples. The expression of both TNF- $\alpha$ and IL- 6 were significantly decreased in TLR $2^{-/-}$, TLR4 ${ }^{-/-}$and TLR9 ${ }^{-/-}$mice compared with WT mice. In addition, the results revealed that knockdown of TLR2, 4 or 9 decreased immune cell infiltration and therefore may attenuate lung injury. Furthermore, the overall survival was significantly increased in TLR $2^{-/-}, 4^{-/-}$and $9^{-/-}$CLP-induced ALI mice compared with the WT CLP-induced ALI mice. However, there was no statistical significance between TLR3 ${ }^{-/}$CLP-induced ALI and WT CLP-induced ALI in the current study. Taken together, these results suggest that in the sepsis-induced ALI model, several TLRs are upregulated and participate in the inflammatory response. Therefore, inhibition of multiple TLRs including TLR2, 9, and especially TLR4 simultaneously, but not TLR3, may be a potential therapeutic target for the treatment of sepsis-induced ALI.
\end{abstract}

Correspondence to: Professor Xiangyan Liu, Department of Thoracic Surgery, Shandong Provincial Hospital Affiliated to Shandong University, 324 Jingwu Road, Jinan, Shandong 250000, P.R. China

E-mail: liuxy6638@126.com

Key words: acute lung injury, sepsis, cecal ligation and puncture, Toll-like receptors, cytokines

\section{Introduction}

Acute lung injury (ALI) can be caused by several factors and imposes a burden on public health, with in-hospital mortality $>40 \%(1,2)$. The overproduction of inflammatory mediators causes endothelial and epithelial injury and induces vascular leakage, edema and vasodilatation, which serve a fundamental role in the pathogenesis of ALI $(3,4)$. Sepsis, which is a leading cause of morbidity and mortality, is the systemic inflammatory response to microbial infection (5). The lungs are particularly vulnerable to the septic inflammatory response, and sepsis is the underlying cause of ALI in most patients (6). Therefore, the identification of therapeutic and preventive approaches that are innovative, safe and effective is crucial for the treatment of sepsis-induced organ injury.

Innate immune cell activation depends primarily on Toll-like receptors (TLRs), a family of pattern recognition receptors (PRRs) that can recognize specific pathogen-associated molecular patterns (PAMPs), which include nucleic acids, proteins and lipids $(7,8)$. TLRs are key regulators of both the innate and adaptive immunity and one of the most well studied PRRs (9). To date, $>10$ members of the TLR family have been identified in mammals, with each receptor recognizing a specific set of PAMPs (10). TLR2 recognizes peptidoglycan and lipoteichoic acid, while TLR4 recognizes lipopolysaccharide (11). TLR3 recognizes double-stranded RNA within endosomes, while TLR9 recognizes unmethylated bacterial CpG-DNA (12-14). TLR3 and 9 are intracellular receptors, while TLR2 and 4 are cell surface receptors (15). However, activation of different TLRs induces similar pro-inflammatory responses with the produces cytokines, which include TNF- $\alpha$ and IL-6 (16). TLRs and their associated signalling pathways constitute an interlaced network, which makes it complicated and difficult to identify novel therapeutic targets (17). Furthermore, inflammation is often caused by multiple PAMPs (18). Therefore, multiple TLRs may be activated in a disease, which adds complexity to potential treatment strategies. It is therefore necessary to identify which TLRs are involved in the process of an inflammatory disease.

Sepsis is one of the leading risk factors for ALI, and there are many animal models of sepsis, which include the administration of live bacteria and bacterial products (19-21). Cecal ligation and puncture (CLP) has become the most widely used model for sepsis, in which the cecum is ligated 
and punctured three to five times with a needle and PAMPs from bacterial products can be released into the blood (22). CLP causes acute lung injury similar to ALI; however, the actual bacterial inoculum in CLP has not been identified (23). Blood cultures from these models are usually positive for specific Gram-positive and Gram-negative bacteria, the components of which can increase the production of multiple inflammatory cytokines by different TLRs, which include IL-6 and TNF- $\alpha$ (24).

Given the results of previous studies, the aim of the current study was to investigate the role of different TLRs during sepsis and sepsis-induced ALI. A CLP-induced ALI model in C57BL/6 WT, TLR2 ${ }^{-/-}$TLR $^{-/-}$, TLR4 ${ }^{-/}$and TLR9 ${ }^{-/}$C57BL/6 mice was established. The current study demonstrated that multiple TLRs may contribute to the pathogenesis of sepsis-induced ALI, and TLRs serve a specific role in this process. Therefore, novel therapeutic targets, which inhibit the activation of multiple TLRs simultaneously, require investigation.

\section{Materials and methods}

Animals. A total of 60 wild-type (WT) and 140 TLR knockout $\left(\mathrm{TLR}^{-/}\right.$), including $\mathrm{TLR}^{-/-}$TLR $^{-/}$, $\mathrm{TLR}^{-/-}$and TLR9 ${ }^{-/}$, C57BL/6 male mice (8-10 weeks; weight, 18-20 g) were obtained from the Chinese Academy of Inspection and Quarantine (Beijing, China). All mice were housed in plastic cages at $20-25^{\circ} \mathrm{C}$, and a relative humidity of $40-70 \%$ under a $12 \mathrm{~h} \mathrm{light/dark} \mathrm{cycle.} \mathrm{All} \mathrm{mice} \mathrm{were} \mathrm{housed} \mathrm{in} \mathrm{the}$ Experimental Animal Centre of the Medical Research Center at the Provincial Hospital Affiliated to Shandong University (Jinan, China) and kept under specific-pathogen-free conditions with free access to food and water. Following subsequent experimental procedures, all mice were euthanized by $\mathrm{CO}_{2}$. All experimental procedures were performed in accordance with the National and Institutional Guidelines for Animal Care and Use and approved by the Institutional Animal Ethics Committee of Shandong University (Jinan, China).

CLP-induced ALI model. C57BL/6 mice were divided into six groups: WT sham, WT CLP, TLR2 ${ }^{-/}$CLP, TLR3 ${ }^{-/-}$CLP, TLR4 $^{-/}$CLP and TLR9 ${ }^{-/}$CLP. CLP was used to establish the ALI mouse model used in the current study. For the CLP procedure, mice were fasted, with only free access to water for $12 \mathrm{~h}$ prior to surgery. Subsequently, mice were anesthetized with 5\% isoflurane (Shanghai Yuyan instruments, Co., Ltd., Shanghai, China) and maintained at $1.5 \%$ isoflurane in $70 \% \mathrm{~N}_{2} \mathrm{O}$ and $30 \% \mathrm{O}_{2}$ using a small animal anesthetic machine (Midmark Corporation, Kettering, OH, USA). Following anaesthesia, skin was disinfected and a median incision was made in the abdomen, allowing the cecum to be exposed and a 4-0 braided silk suture was passed through the midpoint between the colon root and cecum terminal. A 21-gauge 1-inch needle was inserted into the cecum ligation and a small drop of the intestinal content was squeezed to induce infection. Finally, the, cecum was repositioned and the incision was closed. For the sham group, the abdomen was opened, cecum exposed and repositioned, and the incision was closed.

Reverse transcription-quantitative polymerase chain reaction $(R T-q P C R)$. Animals were sacrificed and lungs were isolated at
Table I. Primer pair sequences for quantitative polymerase chain reaction.

\begin{tabular}{ll}
\hline Gene & \multicolumn{1}{c}{ Primer sequence (5'-3') } \\
\hline TLR2 & F:CGATGACTACCGCTGTGACTC \\
& R: CCTTCCTGGGCTTCCTCTT \\
TLR3 & F: GGGACTGTTGACCTGTT \\
& R: GTTGGCTGTATCTCGTAA \\
TLR4 & F: TGCCTTCACTACAGGGACTTT \\
& R: TGGGACACCACGACAATAAC \\
TLR9 & F:CGTGACAATTACCTGGCCTTC \\
& R: CAGGGCCTTCAGCTGGTTC \\
$\beta$-actin & F: ATGAAGATCCTGACCGAGCG \\
& R: TACTTGCGCTGAGGAGGAGC
\end{tabular}

TLR, Toll-like receptor; F, forward; R, reverse.

$20 \mathrm{~h}$ post-surgery. Total RNA was extracted from lung samples using TRIzol ${ }^{\circledR}$ reagent, according to the manufacturer's protocol (Invitrogen; Thermo Fisher Scientific, Inc., Waltham, MA, USA). Total RNA was reverse transcribed into cDNA using the High-Capacity cDNA Reverse Transcription kit (Applied Biosystems; Thermo Fisher Scientific, Inc.). mRNA expression was evaluated by qPCR using the Maxima SYBR Green/ROX qPCR Master Mix 2X (Thermo Fisher Scientific, Inc.) and the Mastercycler ${ }^{\circledR}$ Realplex system (Eppendorf, Hamburg, Germany). Primer pair sequences are summarised in Table I. qPCR quality and genomic DNA contamination was examined using intron-spanning primers, reverse transcriptase-negative samples from cDNA synthesis and melting curve analysis obtained from each reaction. The amplification conditions were as follows: $95^{\circ} \mathrm{C}(10 \mathrm{sec})$, followed by 40 cycles at $55^{\circ} \mathrm{C}(20 \mathrm{sec}), 72^{\circ} \mathrm{C}(25 \mathrm{sec})$. mRNA levels were quantified using the $2^{-\Delta \Delta \mathrm{Cq}}$ method (25) and normalized to the internal reference gene $\beta$-actin. The results are expressed relative to the values of the sham (CLP-induced sepsis) group.

Western blot analysis. Total protein was extracted from lung tissue using a T-PER Tissue Protein Extraction Reagent kit according to the manufacturer's protocol (Thermo Fisher Scientific, Inc). Total protein was quantified using a bicinchoninic acid (BCA) assay kit (Beyotime Institute of Biotechnology, Haimen, China). Samples were then separated on $12 \%$ SDS-Polyacrylamide gel (20 $\mu \mathrm{g}$ per lane) and transferred on to PVDF membranes in an ice bath. After blocking the non-specific site with blocking solution (5\% non-fat dry milk) at room temperature for $2 \mathrm{~h}$, the membrane was incubated overnight at $4^{\circ} \mathrm{C}$ with the following specific primary antibodies: Primary antibodies against TLR2 (1:1,000; cat. no. ab213676; Abcam, Cambridge, MA, USA), TLR3 (1:500; cat. no. ab62566; Abcam), TLR4 (1:500; cat. no. ab13556; Abcam) and TLR9 (1:500; cat. no. ab37154; Abcam). Samples were then incubated with horseradish peroxidase-labelled IgG secondary antibody (cat. no. A0208; 1:2,000; Beyotime Institute of Biotechnology) for $1 \mathrm{~h}$ at room temperature. Chemiluminescent liquid (cat. no. WBKLS0100; EMD 
A

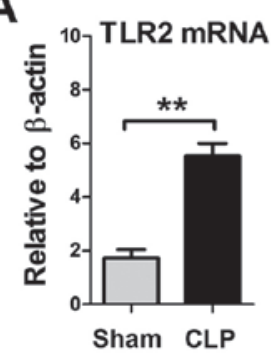

B

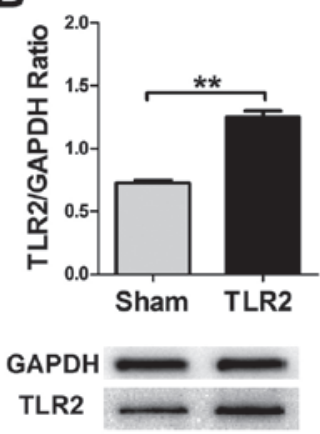

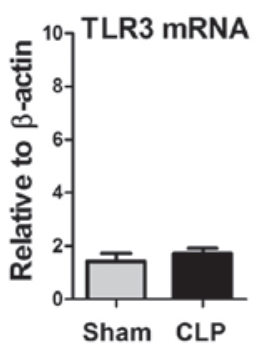
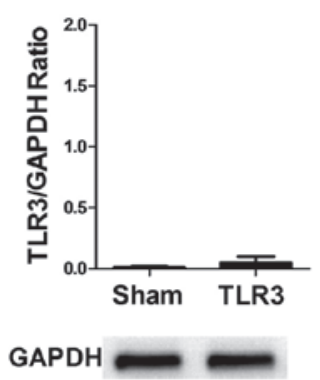

TLR3
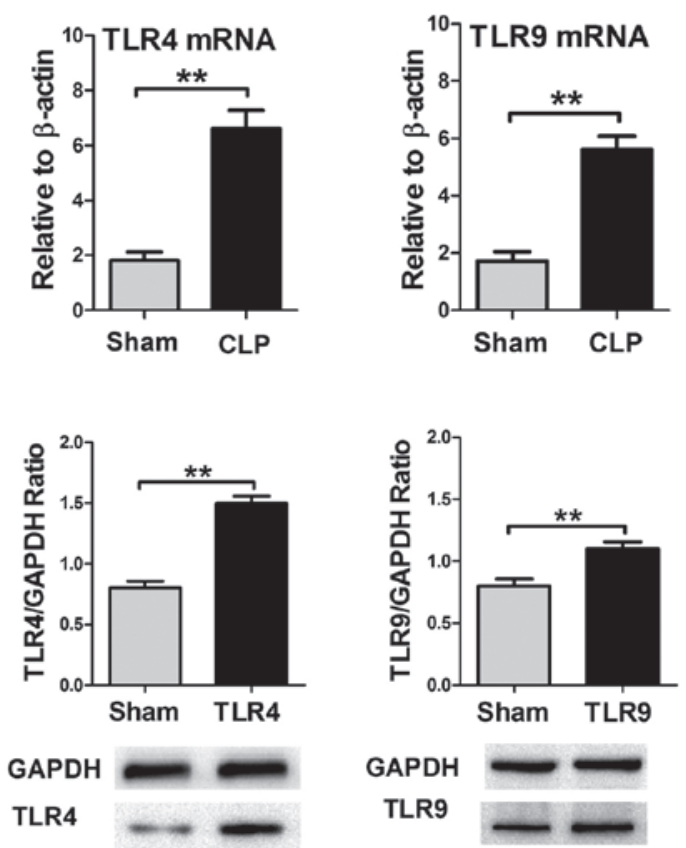

GAPDH

TLR9

Figure 1. TLR2, 4 and 9 mRNA and protein expression levels increase in mouse lung tissue following CLP-induced ALI. WT C57BL/6 male mice were divided into two groups: CLP- and sham-operated groups, and lungs were isolated at 20 h post-surgery. The relative (A) mRNA and (B) protein expression levels of TLR2, 4 and 9 were determined by RT-qPCR and western blot analysis, respectively. Data are presented as the mean \pm standard error of the mean $(n=5)$. ${ }^{* *} \mathrm{P}<0.01$ vs. sham group. TLR, Toll-like receptor; CLP, cecal ligation and puncture; ALI, acute lung injury; WT, wild-type; RT-qPCR, reverse transcription-quantitative polymerase chain reaction.

Millipore) was prepared in a ratio of 1:1. Protein expression was quantified and scanned using the ChemiDoc XRS+ system (Bio-Rad, CA, USA) with GAPDH (1:500; cat. no. ab8245; Abcam) as the loading control. Finally, The band density was quantified using Image J software (v1.8.0; National Institutes of Health, Bethesda, MD, USA).

ELISA. Mouse plasma and lung tissue samples were analysed for TNF- $\alpha$ (cat. no. 88-7324-22) and IL-6 (cat. no. 88-7064-88) expression using ELISA kits obtained from eBioscience (Thermo Fisher Scientific, Inc.), according to the manufacturer's protocol.

Collection of bronchoalveolar lavage fluid (BALF) samples. BALF samples were collected from mice following bronchoalveolar lavage as previously described (26). Briefly, mice were anesthetised and lungs were exposed. The tracheas were cannulated and the lungs were lavaged twice with $0.8 \mathrm{ml}$ normal saline. The BALF samples were collected and centrifuged at $800 \mathrm{x} \mathrm{g}$ for $10 \mathrm{~min}$ at $4^{\circ} \mathrm{C}$, and the supernatant was removed and stored at $-80^{\circ} \mathrm{C}$ until further use. The cell pellet was resuspended and the total number of cells was determined using a hemocytometer. The total number of leukocytes in the BALF sample was counted and classified into five categories including, neutrophils, basophils, eosinophils, macrophages and lymphocytes on a Hemavet 950 instrument (Drew Scientific Inc., Miami Lakes, FL, USA). Total protein in the BALF samples was quantified using a BCA assay kit (Beyotime Institute of Biotechnology).

Haematoxylin and eosin (H\&E) staining. Lung tissue samples from CLP-induced ALI mice at $20 \mathrm{~h}$ were fixed in
$10 \%$ formalin for $\geq 24 \mathrm{~h}$ at $22^{\circ} \mathrm{C}$ and embedded in paraffin. Paraffin-embedded tissue samples were cut into 5- $\mu$ m-thick sections. Tissue sections were subsequently deparaffinized and stained with haematoxylin erythrosine saffron and morphological changes were observed under the light microscope (magnification, x200). Lung injury was scored by two independent pathologists according to the following four items: Alveolar congestion, hemorrhage, infiltration or aggregation of neutrophils in airspaces or vessel walls, and thickness of alveolar wall or hyaline membrane formation. Each item was graded according to a four-point scale: $0=$ minimal damage/appears normal; 1 = mild damage; 2 = moderate damage; $3=$ severe damage; and $4=$ maximal damage. The total lung injury score was calculated by adding up the individual scores for each category, ranging from 0 to 16 (most severe).

Mortality. For survival studies, each mouse was monitored daily for 7 days following CLP or sham surgery. Observations for survival were performed every $24 \mathrm{~h}$ for 7 days.

Statistical analysis. Data are expressed as the mean \pm standard error of the mean. All statistical analyses were performed using SPSS software (version 17.0; SPSS, Inc., Chicago, IL, USA). The independent-sample t-test was used to analyse the statistical difference between two groups. One-way analysis of variance followed by Fisher's least significant difference post hoc test was used to analyse differences among multiple groups. Overall survival was analysed using the Kaplan-Meier method and the log-rank method was used to compare 1-week survival distribution between groups. $\mathrm{P}<0.05$ was considered to indicate a statistically significant difference. 

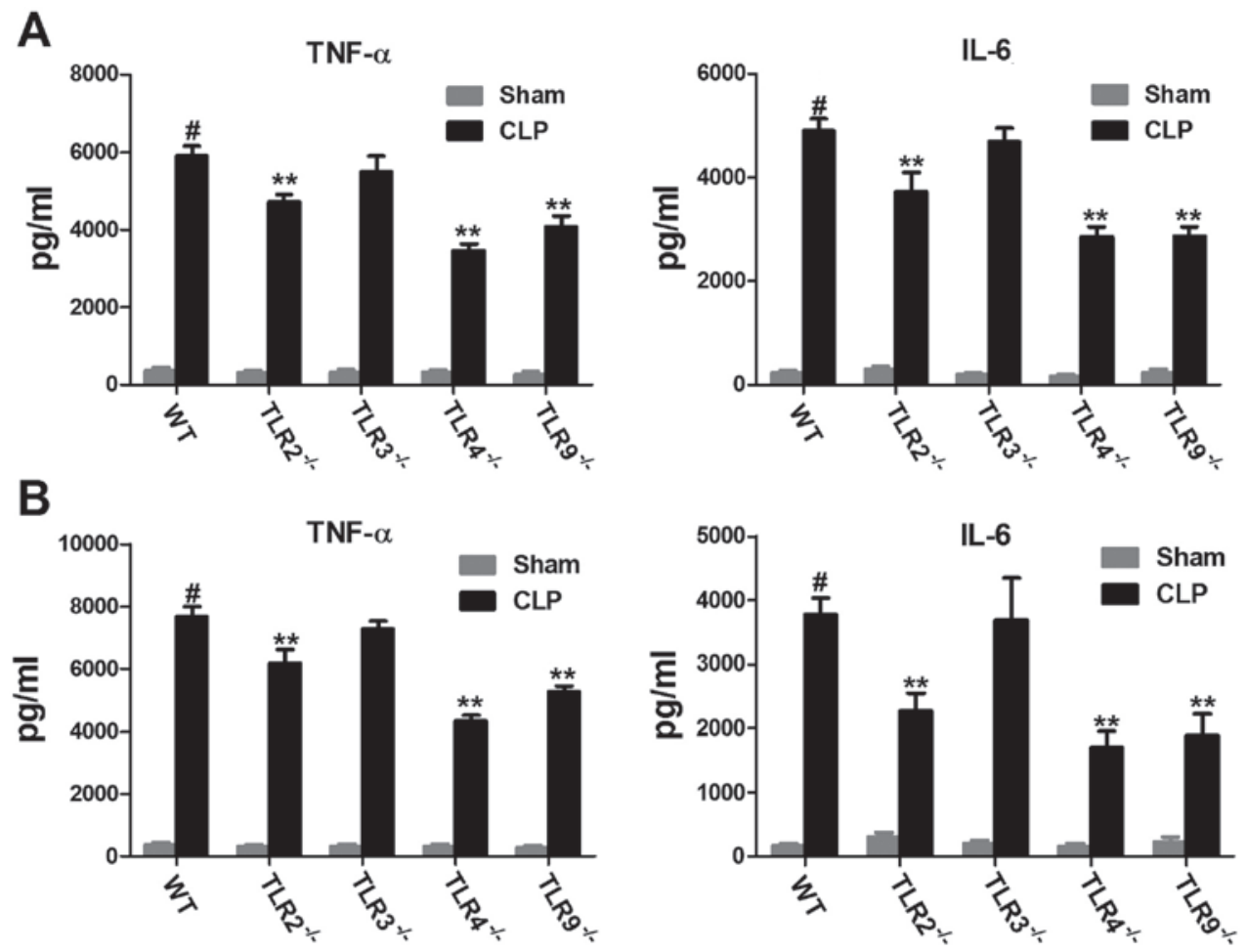

Figure 2. TNF- $\alpha$ and IL-6 expression decreases in CLP-induced ALI in TLR-deficient mice. WT and TLR-deficient C57BL/6 mice received CLP surgery. TNF- $\alpha$ and IL- 6 expression in (A) plasma and (B) lung tissue were examined by ELISA 20-h post-surgery. Data are presented as the mean \pm standard error of the mean $(\mathrm{n}=5) .{ }^{*} \mathrm{P}<0.05$ vs. the WT sham group; ${ }^{* *} \mathrm{P}<0.01$ vs. WT CLP group. TNF- $\alpha$, tumor necrosis factor- $\alpha$; IL-6, interleukin- 6 ; CLP, cecal ligation and puncture; ALI, acute lung injury; TLR, Toll-like receptor; WT, wild-type.

\section{Results}

TLR2, 4 and 9 mRNA and protein expression levels increased in lung tissue following CLP-induced ALI. CLP is the most widely used model of sepsis, whereby multiple PAMPs from bacterial products are released into the blood (27).CLP-induced ALI mode can be used to identify upregulated TLRs, which are regulated by PAMPs. To detect the expression of TLR2, 3, 4 and 9 in CLP-induced ALI, total RNA and protein were isolated from the lung tissue of WT and CLP-induced ALI mice. The mRNA expression levels of TLR2, 4 and 9 were significantly increased in the CLP-operated group compared with the sham-operated group (Fig. 1A). Similarly, increased in the CLP-operated group the protein expression levels of TLR2, 4 and 9 were increased compared with the sham-operated group (Fig. 1B). However, there were no significant changes in the mRNA and protein expression level of TLR3 in the CLP-operated group compared with the sham-operated group (Fig. 1A and B). These results suggest that PAMPs in CLP-induced ALI may induce TLR2, 4 and 9 mRNA and protein expression.

TLR2, 9 and 4 increased cytokine production in CLP-induced $A L I$. To investigate the role of TLRs in the inflammatory response in CLP-induced ALI, the inflammatory response in TLR-deficient and WT mice was examined. Mouse plasma and lung tissue samples were analysed for TNF- $\alpha$ and IL- 6 expression. The expression levels of TNF- $\alpha$ and IL-6 in the plasma of CLP-induced ALI WT mice were significantly increased compared with the sham-operated mice
(Fig. 2A and B). In addition, the expression levels of TNF- $\alpha$ and IL-6 in the plasma of CLP-induced ALI TLR-deficient mice were markedly increased compared with the sham-operated mice (Fig. 2A and B). However, the expression levels of TNF- $\alpha$ and IL- 6 in the plasma of CLP-induced ALI in TLR2-, 4- and 9-deficient mice were significantly decreased compared with CLP-induced ALI WT mice. Furthermore, there was no difference in the expression levels of TNF- $\alpha$ and IL-6 in the plasma of CLP-induced ALI in TLR3-deficient mice compared with CLP-induced ALI WT mice. Similarly, the trend in the expression levels of TNF- $\alpha$ and IL-6 in plasma was also observed in lung tissue samples (Fig. 2B). These results suggest that some TLRs including TLR2, 4 and 9 may increase CLP-induced cytokine production and aggravate ALI, however TLR3 may not.

Knockdown of TLR2, 4 or 9 may attenuate lung injury. The main features associated with CLP-induced lung injury are hypoxemia, immune cell infiltration, as well as interstitial and alveolar edema. In the CLP-induced ALI groups, the total BALF cell count, including, neutrophils and macrophages as well as protein concentration were increased compared with the sham group (Fig. 3A-D). However, compared with the CLP-induced ALI WT mice, the total BALF cell count, as well as BALF neutrophil and macrophage counts were significantly decreased in the CLP-induced ALI TLR2-, 4- and 9-deficient mice (Fig. 3A-C). In addition, the BALF protein concentration was significantly decreased in the CLP-induced ALI TLR2-, 4- and 9-deficient mice compared with the CLP-induced ALI WT mice (Fig. 3D). 
A

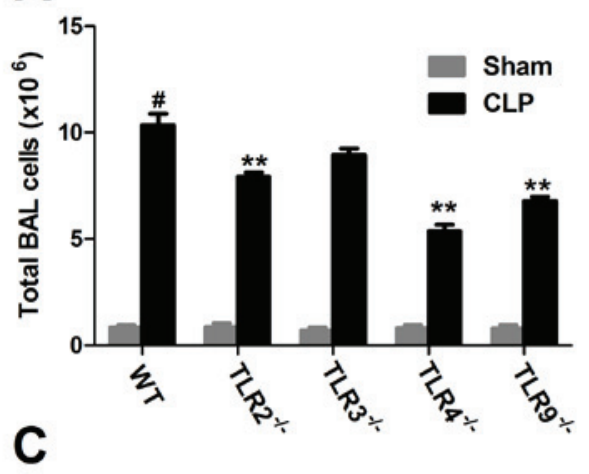

C

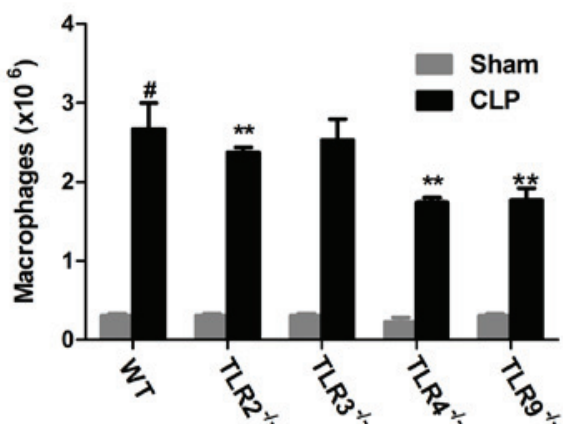

B
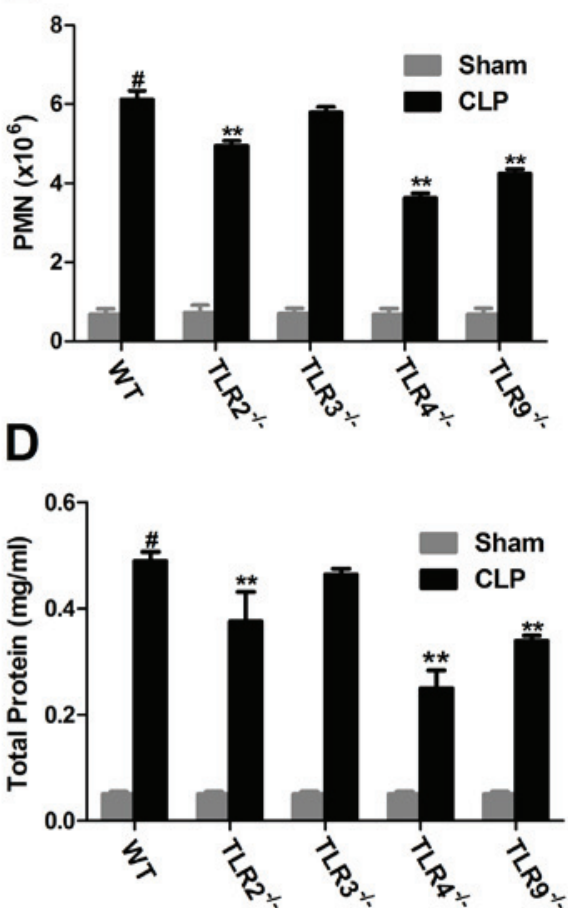
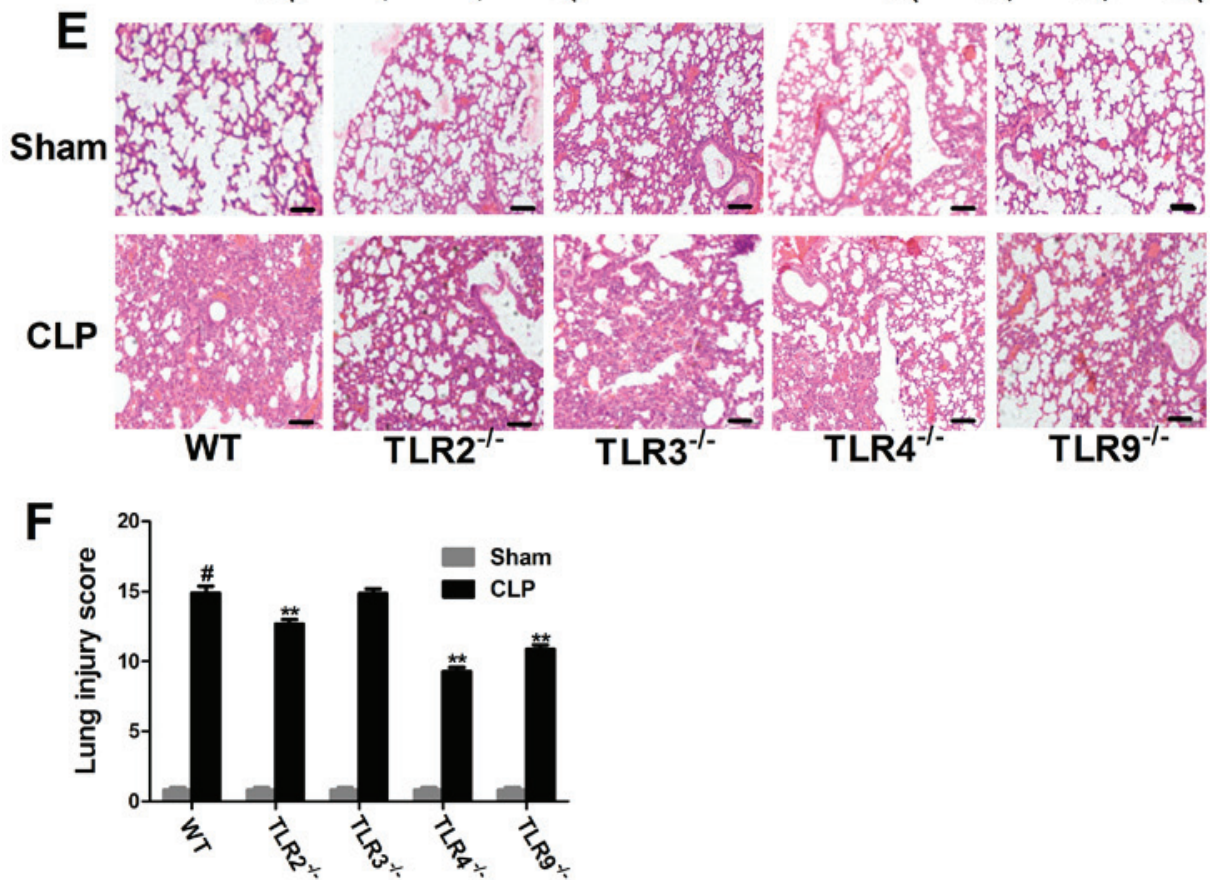

Figure 3. Immune cell infiltration and lung injury in CLP-induced ALI in WT and TLR ${ }^{-/}$mice. BALF samples were collected from WT and TLR ${ }^{-/}$mice 20-h post-surgery and the (A) total, (B) PMN and (C) macrophage count was examined in each group. (D) BALF protein concentration was examined. (E) Morphological changes were observed following H\&E staining in lung tissue sections (magnification, x200). Scale bar, $200 \mu \mathrm{m}$. Data from at least three independent experiments. (F) CLP-induced lung injury scores were examined. Data are presented as the mean \pm standard error of the mean ( $\mathrm{n}=5$ ). ${ }^{*} \mathrm{P}<0.05 \mathrm{vs}$. the WT sham group; " $\mathrm{P}<0.01$ vs. WT CLP group. CLP, cecal ligation and puncture; ALI, acute lung injury; WT, wild-type; TLR, Toll-like receptor; PMN, polymorphonuclear cells; BALF, bronchoalveolar lavage fluid; H\&E, hematoxylin and eosin.

H\&E stained lung sections demonstrated that there were a large number of infiltrating inflammatory cells in the CLP-induced ALI WT group compared with the sham group (Fig. 3E). In addition, the lung injury score was significantly increased in the CLP-induced ALI WT group compared with the sham group (Fig. 3F). However, the number of infiltrating inflammatory cells decreased in the CLP-induced ALI TLR2-, 4- and 9-deficient mice, with a significantly decreased lung injury score compared with the CLP-induced ALI WT group (Fig. 3E and F). By contrast, the pathological injury in the CLP-induced ALI TLR3-deficient mice was not improved, and there was no statistical difference in the lung injury score compared with the CLP-induced ALI group (Fig. 3E and F). Taken together, these results suggest that knockdown of TLR2 and 9, and especially TLR4, may attenuate lung injury in CLP-induced ALI. 


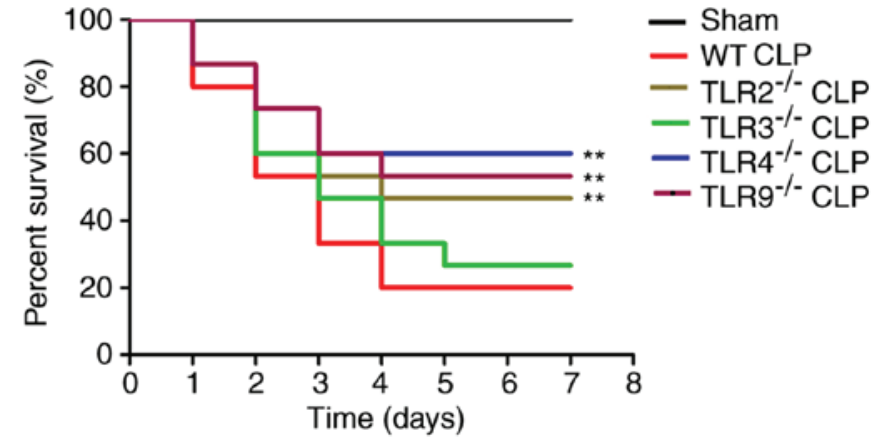

Figure 4. Overall survival of CLP-induced ALI in WT and TLR ${ }^{-/-}$mice. Kaplan-Meier survival curves following CLP-induced ALI in WT and TLR ${ }^{-1}$ mice $(n=15)$. The log-rank method was used to compare 1-week survival distributions among the different groups. ${ }^{* *} \mathrm{P}<0.01$ vs. WT CLP group. WT, wild-type; CLP, cecal ligation and puncture; TLR, Toll-like receptor.

TLR2, 4 and 9 expression increased the mortality of CLP-induced ALI mice. To investigate the effect of TLRs on the mortality in CLP-induced ALI, overall survival was examined in TLR-deficient and WT mice. Overall survival was significantly decreased in the CLP-induced ALI WT group compared with the sham-operated group; however, the overall survival was significantly increased in the CLP-induced ALI TLR2-, 4- and 9-deficient mice compared with the CLP-induced ALI WT mice (Fig. 4). There was no statistically significant difference between the CLP-induced ALI TLR3-deficient mice and the CLP-induced ALI WT mice. These results suggest that TLR2, 4 and 9 increased mortality in the CLP-induced ALI mice.

\section{Discussion}

Although activation of TLRs is essential to the innate immune system and serve a role in the host defensive mechanism against invading microorganisms, overactivation of TLRs is involved in the pathogenesis of several inflammatory diseases (28). Acute respiratory distress syndrome (ARDS) is the most severe form of ALI, which is caused by a severe systemic inflammatory response due to several risk factors including sepsis, major surgery and trauma (29). Severe infection and major trauma induces the systemic release of inflammatory mediators and subsequent indirect lung injury (30).

The innate immune response provides protection against invading pathogens and relies on PRRs to recognize conserved microbial motifs or PAMPs (31). TLRs were one of the first major families of PRRs discovered in mammals, and are expressed on immune cells, including DCs, macrophages and B cells, as well as specific non-immune cells, which include endothelial and epithelial cells (32). In addition, TLRs 1-10 are mainly expressed in lung tissue (33) and individual TLRs can be differentially regulated in specific lung cell populations in response to microbial stimulation (34). The activation of specific TLRs can lead to the expression of several proinflammatory cytokines, including TNF- $\alpha$, IL-6, IL-12 and IFNs (35).

Although a previous study has investigated the relationship between TLRs and sepsis or ALI, the current study is the first to examine multiple TLRs simultaneously and compare their underlying role in sepsis-induced ALI (36). The current study established a CLP-induced ALI mouse model to examine the effect of four TLRs, which included TLR2, 3, 4 and 9, on sepsis-induced ALI. The CLP sepsis model is currently regarded as the gold standard for sepsis-related studies because of its high stability, good repeatability and wide applicability (37). The current study used the CLP-induced ALI mouse model to examine cellular changes in TLR2, 3, 4 and 9 mRNA and protein expression in the process of pulmonary acute inflammatory response. The results of the current study demonstrated that the mRNA and protein expression levels of TLR2, 4 and 9 were significantly increased in the CLP-operated group compared with the sham-operated group, and activation of these TLRs also increased cytokine production and mortality in CLP-induced ALI mice. However, the mRNA and expression protein levels of TLR3 were not significantly affected. Taken together, these results suggest that TLRs 2, 4 and 9 may be involved in the pathogenesis of ALI. In addition, knockdown of TLR2 and 9, and especially TLR4, may attenuate lung injury. In the current study, TLR $2^{--}, 3^{-/}, 4^{-/}$and $9^{-/-}$mice were used to demonstrate that knockdown of TLR2, 4 and 9 significantly increased the expression levels of TNF- $\alpha$ and IL-6, while knockdown of TLR3 significantly decreased the expression levels of TNF- $\alpha$ and IL-6. In addition, the number of infiltrating inflammatory cells decreased in TLR $2^{-/}, 4^{-/}$and $9^{-/-}$CLP-induced ALI mice, with a significantly decreased lung injury score compared with the CLP-induced ALI WT mice. Furthermore, the overall survival was significantly increased in TLR2 $2^{-/}$, $4^{-/-}$and $9^{-/-}$CLP-induced ALI mice compared with the WT CLP-induced ALI mice.

ALI is a major cause of morbidity and mortality in intensive care units, despite improvements in supportive care $(6,38)$. Inhibition of the TLR signalling pathways is likely to be an effective therapeutic target for ALI/ARDS. There are several TLR antagonists, most of which can only inhibit the activation of a single TLR. In addition, current treatment strategies have a limited effect on improving overall survival in patients with ALI/ARDS (39-41). Therefore, novel therapeutic targets, which inhibit the activation of multiple TLRs simultaneously, need to be investigated. The current study is the first to examine multiple TLRs simultaneously and compare their underlying role in sepsis-induced ALI, which may be used to improve the screening of potential therapeutic targets.

In conclusion, the current study demonstrated that the mRNA and protein expression levels of TLR2, 4 and 9 were significantly increased in lung tissue samples of CLP-induced ALI mice. Furthermore, multiple TLRs, including TLR2 and 9, and especially TLR4, significantly increased CLP-induced cytokine production and aggravated ALI. The current study demonstrated that knockdown of TLR2, 4 or 9 may attenuate lung injury and overall survival was significantly increased in TLR $2^{-/-}, 4^{-/-}$and $9^{-/}$CLP-induced ALI mice compared with the WT CLP-induced ALI mice. Taken together, these results suggest that multiple TLRs may contribute to the pathogenesis of sepsis-induced ALI. Therefore, inhibition of multiple TLRs including TLR2, 9, and especially TLR4 simultaneously, but not TLR3, may be a potential therapeutic target for the treatment of sepsis-induced ALI/ARDS. 


\section{Acknowledgements}

Not applicable.

\section{Funding}

No funding was received.

\section{Availability of data and materials}

The datasets used and/or analyzed during the current study are available from the corresponding author on reasonable request.

\section{Authors' contributions}

$\mathrm{XC}$ and $\mathrm{XL}$ conceived the project. $\mathrm{XC}$ and TW designed the experiments. XC, TW and LS performed the experiments. XC analysed the data. XC and XL prepared the manuscript. All authors read and approved the final manuscript.

\section{Ethics approval and consent to participate}

All experimental procedures were performed in accordance with the National and Institutional Guidelines for Animal Care and Use and approved by the Institutional Animal Ethics Committee of Shandong University (Shandong, China).

\section{Patent consent for publication}

Not applicable

\section{Competing interests}

The authors declare that they have no competing interests.

\section{References}

1. Seeley EJ, Matthay MA and Wolters PJ: Inflection points in sepsis biology: From local defense to systemic organ injury. Am J Physiol Lung Cell Mol Physiol 303: L355-L363, 2012.

2. Villar J, Sulemanji D and Kacmarek RM: The acute respiratory distress syndrome: Incidence and mortality, has it changed? Curr Opin Crit Care 20: 3-9, 2014.

3. Matthay MA and Zemans RL: The acute respiratory distress syndrome: Pathogenesis and treatment. Annu Rev Pathol 6: 147-163, 2011.

4. Yuk HJ, Lee JW, Park HA, Kwon OK, Seo KH, Ahn KS, Oh SR and Ryu HW: Protective effects of coumestrol on lipopolysaccharide-induced acute lung injury via the inhibition of proinflammatory mediators and NF- $\mathrm{kB}$ activation. J Funct Foods 34: 181-188, 2017.

5. Miyashita T, Ahmed AK, Nakanuma S, Okamoto K, Sakai S, Kinoshita J, Makino I, Nakamura K, Hayashi H, Oyama K, et al: A Three-phase Approach for the Early Identification of Acute Lung Injury Induced by Severe Sepsis. In Vivo 30: 341-349, 2016

6. Butt Y, Kurdowska A and Allen TC: Acute Lung Injury: A Clinical and Molecular Review. Arch Pathol Lab Med 140: 345-350, 2016.

7. Beutler BA: TLRs and innate immunity. Blood 113: 1399-1407, 2009.

8. Cognasse F, Nguyen KA, Damien P, McNicol A, Pozzetto B, Hamzeh-Cognasse $\mathrm{H}$ and Garraud $\mathrm{O}$ : The Inflammatory Role of Platelets via Their TLRs and Siglec Receptors. Front Immunol 6: $83,2015$.

9. Takeda K and Akira S: TLR signaling pathways. Semin Immunol 16: 3-9, 2004.

10. Akira S and Hemmi H: Recognition of pathogen-associated molecular patterns by TLR family. Immunol Lett 85: 85-95, 2003.
11. Mukherjee S, Karmakar S and Babu SP: TLR2 and TLR4 mediated host immune responses in major infectious diseases: A review. Braz J Infect Dis 20: 193-204, 2016.

12. Chattopadhyay S and Sen GC: dsRNA-activation of TLR3 and RLR signaling: Gene induction-dependent and independent effects. J Interferon Cytokine Res 34: 427-436, 2014.

13. Jelinek I, Leonard JN, Price GE, Brown KN, Meyer-Manlapat A, Goldsmith PK, Wang Y, Venzon D, Epstein SL and Segal DM: TLR3-specific double-stranded RNA oligonucleotide adjuvants induce dendritic cell cross-presentation, CTL responses, and antiviral protection. J Immunol 186: 2422-2429, 2011.

14. Vollmer J and Krieg AM: Immunotherapeutic applications of CpG oligodeoxynucleotide TLR9 agonists. Adv Drug Deliv Rev 61: 195-204, 2009.

15. Blasius AL and Beutler B: Intracellular toll-like receptors. Immunity 32: 305-315, 2010.

16. O'Shea JJ and Murray PJ: Cytokine signaling modules in inflammatory responses. Immunity 28: 477-487, 2008.

17. Tan RST, Ho B, Leung BP and Ding JL: TLR cross-talk confers specificity to innate immunity. Int Rev Immunol 33: 443-453, 2014.

18. Rai V and Agrawal DK: The role of damage- and pathogen-associated molecular patterns in inflammation-mediated vulnerability of atherosclerotic plaques. Can J Physiol Pharmacol 95: 1245-1253, 2017.

19. Matute-Bello G, Frevert CW and Martin TR: Animal models of acute lung injury. Am J Physiol Lung Cell Mol Physiol 295: L379-L399, 2008.

20. Walley KR, Lukacs NW, Standiford TJ, Strieter RM and Kunkel SL: Balance of inflammatory cytokines related to severity and mortality of murine sepsis. Infect Immun 64: 4733-4738, 1996.

21. Altemeier WA, Hung CF and Matute-Bello G: Mouse Models of Acute Lung Injury. In: Acute Lung Injury and Repair. Respiratory Medicine. Schnapp L and Feghali-Bostwick C (eds). Humana Press, Cham, pp5-23, 2017.

22. Dejager L, Pinheiro I, Dejonckheere E and Libert C: Cecal ligation and puncture: The gold standard model for polymicrobial sepsis? Trends Microbiol 19: 198-208, 2011.

23. Zhan J, Liu Y, Zhang Z, Chen C, Chen K and Wang Y: Effect of penehyclidine hydrochloride on expressions of MAPK in mice with CLP-induced acute lung injury. Mol Biol Rep 38: 1909-1914, 2011.

24. Xiang $\mathrm{M}$ and Fan J: Pattern recognition receptor-dependent mechanisms of acute lung injury. Mol Med 16: 69-82, 2010.

25. Livak KJ and Schmittgen TD: Analysis of relative gene expression data using real-time quantitative PCR and the 2(-Delta Delta C(T)) Method. Methods 25: 402-408, 2001

26. Bhandari V, Choo-Wing R, Lee CG, Zhu Z, Nedrelow JH, Chupp GL, Zhang X, Matthay MA, Ware LB, Homer RJ, et al: Hyperoxia causes angiopoietin 2-mediated acute lung injury and necrotic cell death. Nat Med 12: 1286-1293, 2006.

27. Cartwright M, Rottman M, Shapiro NI, Seiler B, Lombardo P, Gamini N, Tomolonis J, Watters AL, Waterhouse A, Leslie D, et al: A Broad-Spectrum Infection Diagnostic that Detects Pathogen-Associated Molecular Patterns (PAMPs) in Whole Blood. EBioMedicine 9: 217-227, 2016.

28. Achek A, Yesudhas D and Choi S: Toll-like receptors: Promising therapeutic targets for inflammatory diseases. Arch Pharm Res 39: 1032-1049, 2016.

29. Kim WY and Hong SB: Sepsis and Acute Respiratory Distress Syndrome: Recent Update. Tuberc Respir Dis (Seoul) 79: 53-57, 2016.

30. Torun AC, Tutuncu S, Ustun B and Akdemir HU: A Study of the Therapeutic Effects of Resveratrol on Blunt Chest Trauma-Induced Acute Lung Injury in Rats and the Potential Role of Endocan as a Biomarker of Inflammation. Inflammation 40: 1803-1810, 2017.

31. Zhu Q, He G, Wang J, Wang Y, Chen W and Guo T: Down-regulation of toll-like receptor 4 alleviates intestinal ischemia reperfusion injury and acute lung injury in mice. Oncotarget 8: 13678-13689, 2017.

32. Medvedev AE, Sabroe I, Hasday JD and Vogel SN: Tolerance to microbial TLR ligands: Molecular mechanisms and relevance to disease. J Endotoxin Res 12: 133-150, 2006.

33. Zarember KA and Godowski PJ: Tissue expression of human Toll-like receptors and differential regulation of Toll-like receptor mRNAs in leukocytes in response to microbes, their products, and cytokines. J Immunol 168: 554-561, 2002. 
34. Yang CS, Lee JS, Rodgers M, Min CK, Lee JY, Kim HJ, Lee KH, $\mathrm{Kim} \mathrm{CJ}$, Oh B, Zandi E, et al: Autophagy protein Rubicon mediates phagocytic NADPH oxidase activation in response to microbial infection or TLR stimulation. Cell Host Microbe 11: 264-276, 2012

35. Chalifour A, Jeannin P, Gauchat JF, Blaecke A, Malissard M, N'Guyen T, Thieblemont N and Delneste Y: Direct bacterial protein PAMP recognition by human NK cells involves TLRs and triggers alpha-defensin production. Blood 104: 1778-1783, 2004.

36. Yu M, Shao D, Liu J, Zhu J, Zhang Z and Xu J: Effects of ketamine on levels of cytokines, NF-kappaB and TLRs in rat intestine during CLP-induced sepsis. Int Immunopharmacol 7 : 1076-1082, 2007.

37. Deng D, Li X, Liu C, Zhai Z, Li B, Kuang M, Li P, Shang S, Song Y, Cen Y, et al: Systematic investigation on the turning point of over-inflammation to immunosuppression in CLP mice model and their characteristics. Int Immunopharmacol 42: 49-58, 2017.
38. Zhou D, Qiu J, Liang Y, Dai W, Yuan D and Zhou J: Epidemiological analysis of 9,596 patients with acute lung injury at Chinese Military Hospitals. Exp Ther Med 13: 983-988, 2017.

39. Creagh-Brown BC, Quinlan GJ,Evans TW and Burke-Gaffney A: The RAGE axis in systemic inflammation, acute lung injury and myocardial dysfunction: An important therapeutic target? Intensive Care Med 36: 1644-1656, 2010.

40. Devaney J, Contreras M and Laffey JG: Clinical review: gene-based therapies for ALI/ARDS: where are we now? Crit Care 15: 224-236, 2011.

41. Staples KJ: Editorial: Therapeutics for acute lung injury: time to call in the DRs? J Leukoc Biol 101: 351-353, 2017.

This work is licensed under a Creative Commons Attribution-NonCommercial-NoDerivatives 4.0 International (CC BY-NC-ND 4.0) License. 